\title{
EFFECTS ON THE ELECTRO-MECHANICAL PROPERTIES OF ANILINE- DOPED POLYESTER FIBRIC
}

\author{
Kogo, A. A. ${ }^{1}$, Ismail, I.M. ${ }^{2}$ and Yakasai, M.Y. ${ }^{2}$ \\ ${ }^{1}$ Department of Polymer and Textile Science, Ahmadu Bello University, Zaria, Nigeria \\ ${ }^{2}$ Department of Chemistry, Saadatu Rimi College of Education, Kumbotso, Kano-State \\ Correspondent E-mail Address: aakogo@abu.edu.ng
}

\begin{abstract}
The effects of Electro-mechanical properties of doped polyester fabric with aniline were investigated. The effects of various concentrations on the electrical as well as mechanical were examined and the percolation threshold was established. Chemical polymerization of aniline on Polyester in hydrochloric acid using potassium dichromate as oxidant was carried out. Polyester based conductive polymer composites were prepared by means of melt mixing with a twin screw. The samples were investigated for electrocoductivity and mechanical properties. The results obtained shows polyester fabric became electrically conductive at a percolation threshold between $1-2.5 \%$ concentrations and also the electrical conductivity increased with the concentration, up to $5.0 \times 10^{-3} \mathrm{~S} / \mathrm{m}$ at $5 \%$ concentration. The tensile characteristics such as average breaking force, work-to-rupture, and \% elongation, of the fabric were all improved. Also Scanning Electron Microscope (SEM) analysis of the samples was studied. Keywords: Doped, percolation, threshold,
\end{abstract} conductivity, elongation

\section{INTRODUCTION}

Since the discovery of intrinsically conductive polymers (ICPs) for which Alan Macdiarmid, Alan Heeger and Hideki Shirakawa won a Nobel Prize in Chemistry in 2000, attempts have been made to introduce electrical conductivity into textile fibres, yarns and fabrics by compositing them with conductive polymers such as polypyrole (PPy), polyaniline (PANI) and polythiophene (PT) (Lekpittaya et al., 2004; Patil and Deogaonkar, 2012). The demand for electrically conductive textiles is growing rapidly not only in relation to industrial needs such as sensing, electromagnetic interference (EMI) shielding, electrostatic discharge, data transfer in clothing, dust and germ-free clothing, corrosion protection, but also for military applications such as camouflage and stealth technology. Polyaniline is outstanding, due to its thermal, environmental and chemical stability, low cost and easy synthesis (Kim et al., 2006; Kutanis et al., 2006). In addition there are reports of PANIcoated or in-situ polymerized non-woven fabrics such as nylon 6, cotton, polyester and Nomex fabrics.

The electrical resistance of the PANI-PET yarns decreased as the concentration of the PANI solution increased. It remained fairly constant up to $55^{\circ} \mathrm{C}$, after which the electrical resistance started increasing with increase in temperature. And, like a typical conductor, the longer the yarn length, the higher the electrical resistance. The modulus and other tensile properties also improved. In all, the conductive yarns showed good environmental stability, and the strength and flexibility were preserved. Wu et al., (2009) used a molecular template (a sulphonated polyaniline) to facilitate integration of a complementary conductive polymer (polyaniline) into wool-based textiles. The efficiency of the polymerization/coating process was enhanced because the template localized the reaction within the textile substrate. The presence of the molecular template resulted in the formation of an adherent, uniform and stable conducting polymer layer. The integration of PANI also improved the electrochemical reversibility of the coating. The coated textile material was suitable for use in biomedical monitoring. Neelakandan et al., (2009) polymerized PANI on woven polyester fabric samples (plain, twill and satin). Each polymerization cycle represented one coating, and as the number of coatings was increased, more of the conducting polymer was deposited on the fabric, forming a stable, adherent coating on the textile substrate; this also caused an increase in \% weight add-on as well as fabric thickness. This was clearly demonstrated by SEM observations. The resistivity can be varied from an antistatic level $\left(10^{10} \Omega \mathrm{cm}\right)$ to a high conductivity level $(10 \Omega \mathrm{cm})$ by varying the concentration of the oxidant and dopant.

The aim of this study is to introduce electrical conductivity onto polyester fabric using aniline as dopant, so as to investigate the effects of Electromechanical properties of the fabric.

\section{MATERIALS AND METHODS}

\section{Materials}

Plain woven, $171 \mathrm{~g} / \mathrm{m}^{2} 100 \%$ Polyester (Dacron) was obtained from the Polymer and Textile Science Departmental store Ahmadu Bello University Zaria. Nigeria, Primasol (NMB), soda ash, Triton X-100, acetone, aniline, potassium dichromate, hydrochloric acid and dimethyl formamide (DMF) were all obtained from Sigma-Aldrich. Instruments used include Scanning Electron Microscope (SEM), Instron Tensile testing machine, Water bath sonicator, Probe sonicator and Electrical conductivity. 


\section{Methods}

\section{Fabric Scouring}

The polyester fabric was scoured at $100^{\circ} \mathrm{C}$ for 30 minutes in a bath containing $2 \mathrm{~g} / \mathrm{I}$ non-ionic surfactant (Primasol NMB) and $2 \mathrm{~g} / \mathrm{l}$ soda ash. It was rinsed copiously with water and dried.

\section{Doping of the Fabric}

In a $250 \mathrm{ml}$ flat-bottomed flask $0.3 \mathrm{M}$ aniline (monomer) was stirred in $0.25 \mathrm{M} \mathrm{H}_{2} \mathrm{SO}_{4}$ for 10 minutes. $1 \mathrm{~g}$ of the fabric was impregnated in this solution at room temperature for 30 minutes with mild agitation. Potassium peroxydisulphate was added slowly to initiate polymerization. Polymerization was allowed for $3 \mathrm{hrs}$ and then the fabric was removed and washed with aqueous $0.25 \mathrm{M} \mathrm{H}_{2} \mathrm{SO}_{4}$ in a beaker (to remove loose polymer, unconsumed monomer and oxidant). The residue washed with deionized water before drying.

\section{Tensile Strength Test}

Instron automatic testing machine with an IBM computer interface and printer according to the ASTM

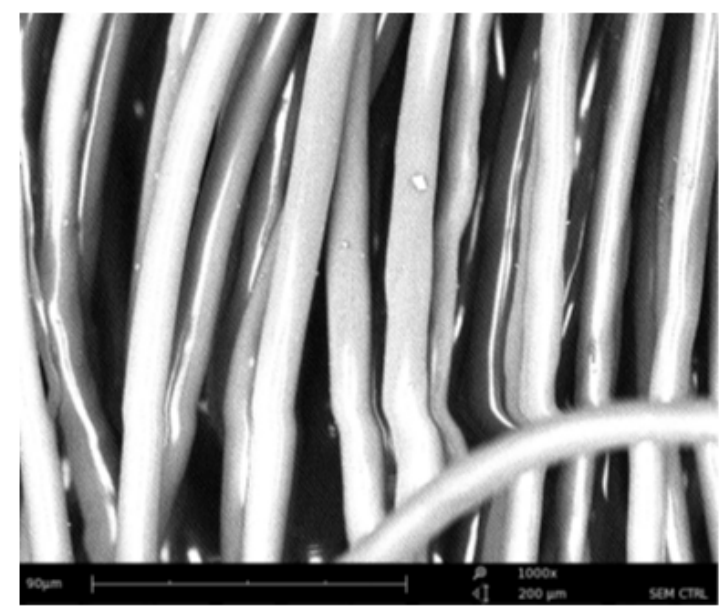

Figure 2: SEM micrograph of the control sample.

Figure 3, shows the relationship of the conducting sample as the function of doped concentration. It can be observed that there is a sharp increased in conductivity between $2.5 \%$. and $3.5 \%$., This region highlighted the percolation threshold. It is the critical concentration above which electrical conductivity
International D 5035-11 (2013), was used to investigate the tensile characteristic such as; average breaking force, percent elongation at break, work of rupture.

\section{Electrical Conductivity Measurement}

METEX auto/manual range dual display digital multimeter with interface (M-3860D) was used to measure the resistance between two probes separated by a known distance.

\section{Results and Discussion}

Figures 1 and 2 are micrographs of control polyester sample and polymerized aniline on the fabric respectively. The Polyaniline deposited as a spongy microfilm within the fabric (Figure2) was observed to have actually penetrated into the filaments, and of course, a larger percentage can be seen coating the filaments and also lodged between their interstices which functions as the electrically conductive layer (ECL).. This corroborates the results from the work of Fugetsu et al., (2009).

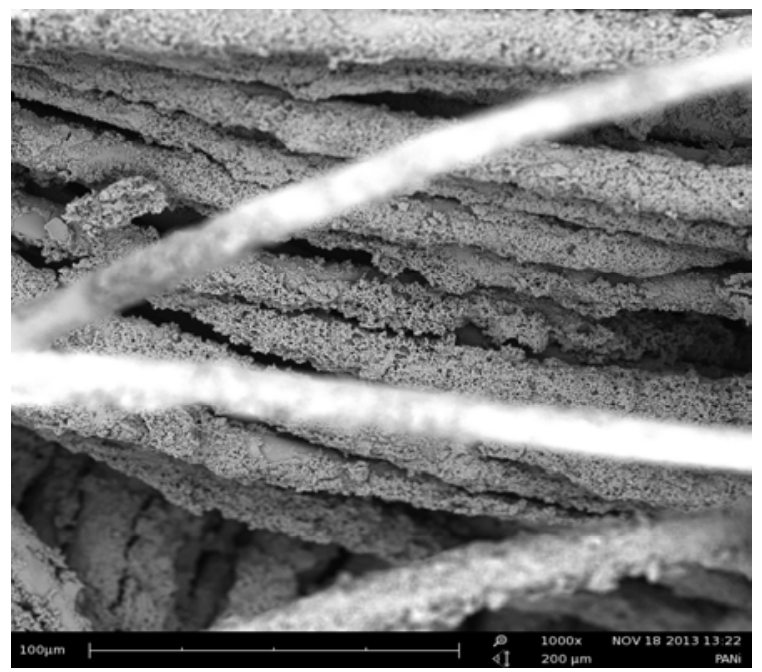

Figure 2: SEM micrograph of polymerized aniline on the fabric (magnified X 1000)

started manifesting in the fabrics. After the percolation threshold, conductivity increased with increase in the concentration, up to a maximum of 5.5 $\%$ after which the fabric ceases to absorbed more (Grossiord et al., 2008).

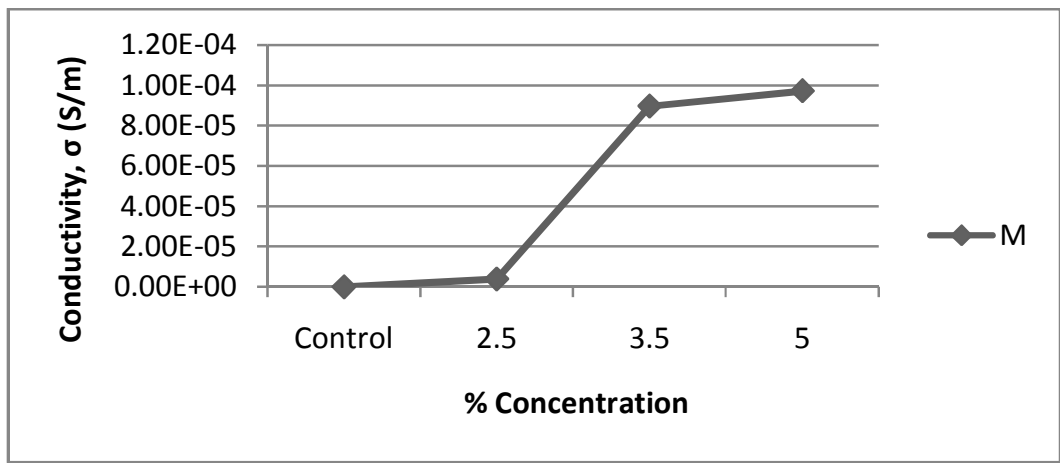

Figure 3: Conductivity, $\sigma$, as a function of $\%$ concentration of aniline 
Fig. 4, shows the plot of average breaking force against \% concentration. There was an increase in average breaking force with increase in concentration, this is ascribed to the difference in the compactness of the yarns, brought about by the interaction of twist levels and number of filaments per cross section of the yarns (Mun et al., 2008).



Figure 4: Average breaking force as a function of \% concentration

Figure 5 and 6 , show the plots of $\%$ elongation and work of rupture versus concentrations, which depicted that they increased with the increased in concentration. Applying a lubricant between the filaments in this way, when a load is applied at the two extremes of a fabric piece, the filaments tend to first straighten out, then untwist, then slide against each other as they attempt to resist stress-bearing
This explains why there was an increase in average \% elongation and work of rupture .The $3.5 \%$ concentration abnormal behaviour were observed because, it is the critical concentration above which the tensile strength started manifesting in the fabrics After the percolation threshold, tensile strength decreased with increase in the concentration (Favini et al.,2012).

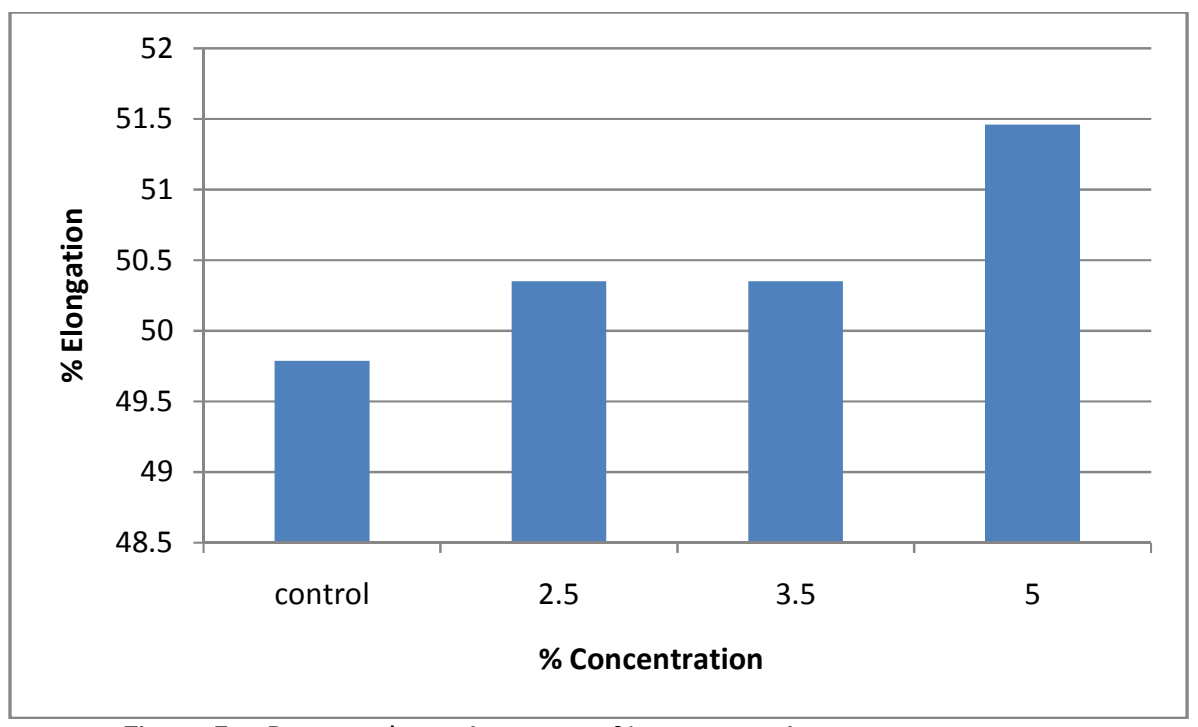

Figure 5: Percent elongation versus \% concentration. 
BAJOPAS Volume 10 Number 1 June, 2017

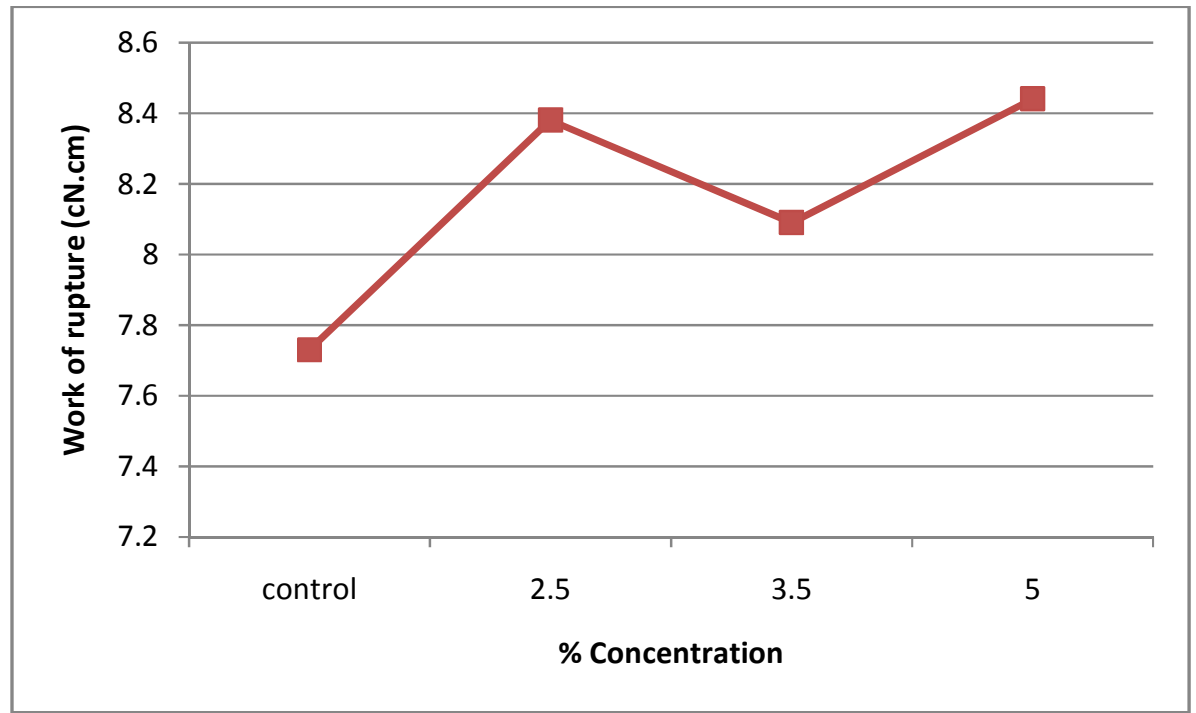

Figure 6: Work of rupture as a function of \% concentration

\section{Conclusion}

Based on its higher yield, aniline was adopted as the intrinsically conductive polymer of choice for our purpose. As it was being polymerized within the fabric, it passed through some landmark, as the dispersion increased the conductivity of the doped

\section{REFERENCES}

ASTM International D1776-08e1 standard practice for conditioning and testing textiles. (www.astm.org , Accessed $30^{\text {th }}$ October 2013)

Favini, E., Agnihotra, S., Surwade, S. P., Niezrecki, C., Willis, D., Chen, J., Niemi, E., Desabrais, K., Charette, C. and Manohar, S.K. (2012): Sensing performance of Electrically Conductive Fabrics and suspension lines for Parachute systems. Journal of Intelligent Material Systems and Structures, 23(17), pp. 1969-1986.

Fugetsu, B., Akiba, E., Hachiya, M., Endo, M. (2009): The Production of Soft, Durable and Electrically Conductive Polyester multifilament Yarns by Dye-printing them with Carbon Nanotubes, Carbon, 47, (2), pp. 527-530

Grossiord, N., Kivit, P. J. J., Loos, J., Meuldijk, J., Kyrylyuk, A.V., van der Schoot, P. and Koning, C. E. (2008): On the Influence of the Processing Conditions on the Performance of Electrically Conductive Carbon nanotube/Polymer nanocomposites. Polymer, 49, pp. 2866-2872. DOI:10.1016/j. polymer. 2008.04.033

Kim, B., Koncar, V. and Dufour, C. (2006): Polyaniline-Coated PET Conductive Yarns: Study of Electrical, Mechanical, and ElectroMechanical Properties. Journal of Applied Polymer Science, Vol. 101, 1252-1256. DOI 10.1002/app.22799

Kutanis, S., Karakışla, M., Akbulut, U. and Saçak, M. (2006): The conductive polyaniline/poly(ethylene terephthalate) fabric was found to vary in a similar way as that of metals i.e., the resistivity or conductivity varies with the length of the conductor and tensile characteristics such as average breaking force, work-of-rupture and $\%$ elongation, of the fabric were all improved.

composite fabrics. Composites: Part A 38, pp 609-614

Lekpittaya, P., Yanumet, N., Grady, B. P. and O'Rear, E. A. (2004): Resistivity of Conductive Polymer-Coated Fabric. Journal of Applied Polymer Science, 92, pp. 2629-2636.

Mun, S.J., Jung, Y.M., Kim, J. and Chang, J. (2008): Poly(ethylene terephthalate) Nanocomposite Fibres with Functionalized Multiwalled Carbon Nanotubes Via In-Situ Polymerization. Journal of Applied Polymer Science, 109, pp 638-646. DOI: 10.1002/app.28164.

Neelakandan, R., Giridev, V. R., Murugesan,M. and Madhusoothanan, M. (2009): Surface Resistivity and Shear Characteristics of Polyaniline Coated Polyester Fabric. Journal of Industrial Textiles, 39, (175). DOI: $10.1177 / 1528083709103315$

Patil, A.J. and Deogaonkar, S.C. (2012 ): A novel Method of in-situ Chemical Polymerization of Polyaniline for Synthesis of Electrically Conductive Cotton Fabrics. Textile Research Journal,82, $1517 . \quad$ DOI: $10.1177 / 0040517512452930$

Wu, J., Zhou, D., Looney, M.G., Waters, P.J., Wallace, G.G. and Too, C.O. (2009): A Molecular Template approach to Integration of Polyaniline into textiles. Synthetic Metals 159, pp. 1135-1140. Retrieved from www.elsevier.com/locate/synmetnt 